\title{
Problematic Quantifications: a Critical Appraisal of Scenario Making for a Global 'Sustainable' Food Production
}

\author{
Andrea Saltelli ${ }^{1,2,3}(\mathbb{D}) \cdot$ Samuele Lo Piano ${ }^{1}$
}

Accepted: 4 August 2017 / Published online: 15 August 2017

(C) Springer International Publishing AG 2017

\begin{abstract}
Over the course of human history food security has represented a primary challenge for civilizations and societies. In the light of the projected trends of population expansion in the forthcoming decades, its primary importance in the global agenda has never decreased. Our contribution to this debate comes in the form of a critique of a paper recently published in the literature, Badur et al. (2016). In their work, the authors suggest that continuous improvements in agricultural techniques and dietary re-adaptation and change will lead in the near future (2050) to a reduced use of land to meet human nutritional needs, even when factoring in a projected human population of 10 billion people. We show that the quantification rests on dubious hypotheses, at odds with present understanding from the field of system ecology, and neglects the core issue that resides in fundamental asymmetries in the food distribution between rich and poor countries. Thus, a political problem is reframed as a technical one, by mobilizing crisp numbers and analytic prowess to convey an impression of prediction and control. We warn that this might veil important underlying ethical issues.
\end{abstract}

Keywords Food security · Sustainability · Sensitivity auditing · Quantitative storytelling · Food distribution $\cdot$ Ecosystem alteration

Andrea Saltelli

andrea.saltelli@uib.no

European Centre for Governance in Complexity (ECGC), Barcelona, Spain

2 Centre for the Study of the Sciences and the Humanities (SVT), University of Bergen (UIB), Bergen, Norway

3 Institut de Ciència i Tecnologia Ambientals (ICTA), Universitat Autonoma de Barcelona (UAB), Barcelona, Spain 


\section{Introduction}

A recent paper entitled 'Pathways Leading to a More Sustainable and Healthy Global Food System' (Badur et al. 2016) presents an analysis of a possible scenario for the interplay between agriculture and world nutritional needs.

The analysis takes the form of a hypothetical executive summary from an imagined report from the Food and Agriculture Organization (FAO). The subject of the report is the state of the world's food systems, written from the perspective of the 2050s.

The study assumes that the world population will have attained 10 billion people, and that agriculture will require " 438 million hectares less land than it did in 2015 ". According to the report this improvement owes much to technological developments, including the application of big data analytics to farming systems in the 2010s and 2020s. Even larger, according to the authors, is the effect of a change of consumption pattern.

The authors imagine a marked shift from the 2015 situation (we recall that the piece is written from the 2050 viewpoint), characterized as systematically overproducing cereals and starches, oils and fats, and sugars, coupled with a lack of $2 / 3$ of amounts of the fruits and vegetables needed for everyone to enjoy a nutritious diet. The authors also refer to a previous (e.g. present day) UN estimate that concludes that there were possibly no lack calories: 3000 dietary calories per person per day were (are hence today) available on the planet. The consequences of overproduction of cereals, oils and sugars were type II diabetes, obesity, and an overuse of agricultural land.

The authors imagine hence that in the intervening period between present day and $2050 \mathrm{a}$ set of virtuous policies have managed to improve the situation. The policy package includes:

1. Consumer Education, in the form e.g. of better food literacy and cooking skills

2. Policies Aimed at Increasing the Cost of Unhealthy Food, including e.g. a "junk food tax"

3. Policies Geared at Capturing the Hidden Environmental Costs Associated with Farming. This foresees a comprehensive market for carbon in order to limit greenhouse-gas emissions from agriculture as well as restrictions on the use of antibiotic use to limit livestock production.

4. A Reduction in the American Corn Subsidy. This policy also aims to raise the price for livestock feed, and for products which contribute to the processed food industry such as high-fructose corn syrup and corn-starch.

5. Enhanced Storage and Processing Facilities in the Developing World. Unlike the previous measures, this specifically targets the 'Global South'.

In this contribution we intend to present a critique of this work. The theoretical framework underpinning our appraisal borrows from the tradition of system ecology (Odum 1968), as well as from the more recent methods of sensitivity auditing (Saltelli and Funtowicz 2014) and quantitative storytelling (Saltelli and Giampietro 2016).

\section{Discussion}

The Authors' bottom line is that "agriculture requires 438 million hectares less land than it did in 2015". 
This 438 Mha figure was arrived at by assuming that:

1. Agriculture shifts away from over production of cereals, oils, and sugars, but increases fruit and vegetables;

2. Agricultural yields increase $\sim 1 \% / \mathrm{y}$ between now and 2050 .

3. Protein consumption shifts from $86 \%$ animals and $14 \%$ plants to $50 \%$ animal and $50 \%$ plant.

While the paper notes that the authors can be contacted for references pertaining to the calculations we feel that we have enough ingredients on the table to sketch a critique of the analysis.

a) How can the hectares extension be given with three significant digits (438 millions)? The many assumptions feeding into the analysis would probably suggest a one digit precision. The most comprehensive database currently available, the FAOstat metadata (FAO 2017), see http://fenixservices.fao.org/faostat/static/documents/RL/RL_e.pdf) warns about the accuracy of the available data due to the lack of consistency and accounting method across countries. A quantification of the error and of the uncertainty in the output would have been appropriate.

b) What percentage decrease of the available land does this number corresponds to: 5 ? $10 \%$ ? A quick look at the sources tells us that total agricultural land in the world amount to some 4.9 billion hectares (FAO, 2014 datum). This would imply a 9\% reduction in land use but this should have been made clear in the article out front.

c) Assuming it refers to an average figure, a $1 \%$ increase in yield over the 35 years separating the authors from the 2050 gives a total increase in yield of $\mathrm{e}^{0.35}=1.4$. Applying this increased yield to the reduced area computed in (b) gives an overall increase in output of about 1.3.

d) Population has been assumed to increase from 7.4 billion (in the year 2015) to 10, corresponding to a factor of 1.3 . Thus, the increase in output would merely balance the increase in population. This would assure the same average food availability at the global level as per today.

e) Assumption (c) was derived assuming a growth rate of about $2 \%$ in the period between now and 2050, decreased to $1 \%$ as per the effect of climate change. One should perhaps also consider the principle of diminishing return, whereby the more efficiency needs to be increased, the more the increment becomes arduous to achieve (Byerlee 1992; Tilman et al. 2002; Struik et al. 2014).

f) The assumption of a less caloric diet is questionable. The authors appear to neglect the expected shift in the population composition by the year 2050. The population projected for the year 2050 will present a higher share of adults given the forecast reduction in natality (Tobergte and Curtis 2013). As adults have higher caloric dietary requirements than children, an overall decrease in cereal production is questionable.

g) The present trend sees a growing meat consumption in a regime of increasing income in developing countries (Smil 2013). Thus, the worldwide desirability (see below) of a change of course is dubious (Gomiero 2016).

h) The effects on the ecosystem of the assumed yield increases are neglected. It is hard to imagine that such agricultural intensification would lead to a decreasing environmental 
pressure, rather a significantly higher ecosystem alteration is foreseeable (Giampietro et al. 1992).

i) The role of legislation and education is emphasized in the article as a valid strategy to achieve the change in the patterns of consumption advocated in this piece. We doubt that a so-called 'food literacy' plan would work on a planetary scale. Exactly as for the case for smoking - mentioning in the piece as an example - a decrease in some developed countries corresponds to an increase in developing ones. Also to be considered are the profound differences in cultural values, and the fact a significant share of the world population in developing countries live under fragile regulatory systems. Even at the national scale, the elaboration of an effective policy is hampered by the disparities in income, ethnicity and age, i.e. the diversity of the people a society is made of. The effectiveness of the campaigns promoting fruit-and-vegetable-based healthy diets has shown to be limited in spite of the consistent resources allocated (Rekhy and McConchie 2014). Finally, the role of possible agricultural-sector lobbies should have been better discussed at the planetary level.

Finally two additional technical points are made:

j) The definition and metering of 'fruit and vegetable intake' is also affected by cultural and ethnic values that make the comparison semantically ambiguous and less robust across studies from different areas and on different societal groups. Different ethnic groups tend to address in different ways the survey questions related to dietary intakes as reported by (Roark and Niederhauser 2013). Is potato intake also in the form of French fries to be considered as vegetable intake? How about onion and tomato slices as well as lettuce when used in small portion as condiments? Perhaps a clear definition of fruit/vegetable serving would be required as this affects the current accounting practices, as well as the proposed policy goals.

k) The paper builds on the postulate that the system can smoothly readjust to its change and seems to neglect possible inelasticity. For instance, the investment required (Demsetz 1969) for a massive production shift from cereals, plant oils and so forth towards vegetables seems to have been neglected. Furthermore, no lowering factor for yields is adopted to take into account the readjustment time.

In summary this study is poorly framed as the problem of today - and likely the challenge of tomorrow as well -is that food is unequally distributed (Falconí et al. 2017). When assessed per kilogram of body mass, caloric intake is not lower in developing countries in comparison with developed countries due to the higher share of children in the former (Giampietro et al. 2011). A higher share of vegetables and fruit in the diet can be certainly desirable worldwide, but we doubt that this can be implemented at the cost of reducing cereal cultivation. The optimistic imprint of the article - which identifies a problem and proceeds to offer its solution has been achieved at the cost of selecting a dubious frame, where food production is unbalanced, too much land is being used and agricultural production can be made more intensive. The article does not offer an estimate of the different balance of fertilizers and pesticides needed in the new scenario, an aspect that would be essential for a thorough assessment of the feasibility and viability (see below) of the proposed transition. An assessment of water use should have been produced as well. As water is primarily withdrawn and used in agriculture (Mekonnen and Hoekstra 2016; Dalin et al. 2017) its local scarcity could 
pose significant challenges for an effective coexistence of agricultural activities with other economic activities.

Contrasted with the global injustice of nutritional inequality we find the policy mix and the inference of the piece appear possibly unethical, determined as it is by a developed world perspective.

This is evidently the substitution of a political problem with a technical one, whereby the world's nutritional unbalances are not the results of a power asymmetry, but of a mere "mismatch between what the world needed for everyone to enjoy a nutritious diet and what the world was actually producing". Additionally, the use of crisp numbers - even in the avowed context an imaginary report - convey a spurious impression of accuracy, which could be termed as rhetorical.

\section{Conclusions}

Our reading of the analysis presented by Badur et al. (2016) has highlighted technical and normative issues.

A major technical issue is that a higher yield from a given amount of land can only be obtained by increasing the external inputs in the agricultural process along with the ecosystem disturbance. This is due to the fact that the biomass grown and taken away is not available for the ecosystem reproduction and this can only be accomplished at the price of linearizing the nutrient flows - i.e. by neglecting the need for a fund - the agricultural land - to regenerate itself in the process (Giampietro et al.2014).

Sensitivity auditing (Saltelli and Funtowicz 2014) and quantitative storytelling (Saltelli and Giampietro 2016) suggest looking at modelling at quantification with an eye to the explicit and implicit frames and assumptions, and to submit those to a reality check based on available data and theory, with a parsimonious use of models and numbers. Thus, we employed a form of sensitivity auditing when checking the assumptions of the Badur et al. (2016) study, while the concept of desirability - whether a given policy option can garner support from the intended recipients, comes from quantitative story-telling. The same holds for feasibility - intended as a compatibility with biophysical constraints, and viability, intended as compatibility with societal internal constraints.

More in general we find that the style of analysis of Badur et al. (2016) implies the replacement of a political and ethical question with a technical one.

Already in 1971 philosopher Jerome R. Ravetz (Ravetz 1996) noted that one of the main difficulties which plagued science as applied to the solution of practical problems is a natural slippage into technical ones (p. 342), oblivious of the associated social dimensions and power relationships.

On the opposite side of this debate it is not rare to see science's advocates attribute the lack of progress to the lay public's obscurantism and lack of understanding of science (Saltelli and Funtowicz 2017). This latter stance is commonly known as deficit model (Wynne 1993), which we consider as unhelpful as a tool to explain the problematic relations between science and society (Saltelli and Funtowicz 2017).

Ravetz further notes (p. 398) "The function of such [technical] explanations is to minimize the threat displayed by the problem-situation; this is done either by explaining it away altogether, or, if this is impossible, to give assurance of the possibility of its comfortable resolution". 
Ravetz's insight is particularly appropriate in light of the ongoing discussion about sustainability of food systems - a central topic of the 2030 Agenda, which will guide decision and policy making in the years to come. Sustainability will need a hard look at power relationships, beyond the comfort of technical solutions.

Acknowledgments The authors would like to thank the anonymous reviewers for their helpful and constructive comments that greatly contributed to improving the manuscript. The responsibility of any error in the manuscript remains solely with us. The research did not receive any specific grant from funding agencies in the public commercial, or not-for-profit sectors.

\section{References}

Badur, Krishna K.C., Evan D.G. Fraser, Samantha Pascoal, Goretty Dias, and Trudi Zundel. 2016. Pathways leading to a more sustainable and healthy global food system. The Solutions Journal 7 (5): 10-12.

Byerlee, Derek. 1992. Technical change, productivity, and sustainability in irrigated cropping systems of South Asia: Emerging issues in the post??? Green revolution era. Journal of International Development 4: 477496. doi:10.1002/jid.3380040502.

Dalin, Carole, Yoshihide Wada, Thomas Kastner, and Michael J. Puma. 2017. Groundwater depletion embedded in international food trade. Nature 543. Nature publishing group: 700-704. doi:10.1038/nature21403.

Demsetz, Harold. 1969. Information and efficiency: Another viewpoint. Journal of Law and Economics 12: 122. doi: $10.1086 / 466657$.

Falconí, Fander, Jesus Ramos-Martin, and Pedro Cango. 2017. Caloric unequal exchange in Latin America and the Caribbean. Ecological Economics 134: 140-149. doi:10.1016/j.ecolecon.2017.01.009.

FAO 2017. Faostat http://www.fao.org/faostat/en/

Giampietro, Mario, Aspinall, Richard, J., Jesus Ramos-Martin, and Sandra G. F. Bukkens. 2014. Resource accounting for sustainability assessment: the nexus between energy, food, water and land use. New York: Routledge.

Giampietro, Mario, Giovanni Cerretelli, and David Pimentel. 1992. Energy analysis of agricultural ecosystem management: Human return and sustainability. Agriculture, Ecosystems \& Environment 38: $219-244$. doi:10.1016/0167-8809(92)90146-3.

Giampietro, Mario, Kozo Mayumi, and Alevgül H. Sorman. 2011. The metabolic pattern of societies: Where economists fall short. Routledge Studies in Ecological Economics. Taylor \& Francis.

Gomiero, Tiziano. 2016. Agriculture and degrowth: State of the art and assessment of organic and biotech-based agriculture from a degrowth perspective. Journal of Cleaner Production. Elsevier Ltd. doi:10.1016/j. jclepro.2017.03.237.

Mekonnen, Mesfin M., and Arjen Y. Hoekstra. 2016. Four billion people facing severe water scarcity. Science Advances 2: e1500323. doi:10.1126/sciadv.1500323.

Odum, Eugene P. 1968. Energy Flow in Ecosystems: A Historical Review. American Zoologist 8. Oxford University Press: 11-18. doi:10.1093/icb/8.1.11.

Ravetz, Jerome R. 1996. Scientific knowledge and its social problems. New Jersey: Transaction Publishers.

Rekhy, Reetica, and Robyn McConchie. 2014. Promoting consumption of fruit and vegetables for better health. Have campaigns delivered on the goals? Appetite 79: 113-123. doi:10.1016/j.appet.2014.04.012.

Roark, Randall A., and Victoria P. Niederhauser. 2013. Fruit and vegetable intake: Issues with definition and measurement. Public Health Nutrition 16: 2-7. doi:10.1017/S1368980012000985.

Saltelli, Andrea, and S.O. Funtowicz. 2014. When all models are wrong. Issues in Science and Technology 30: 79-85.

Saltelli, Andrea, and Silvio Funtowicz. 2017. What is science's crisis really about? Futures. Elsevier Ltd: 1-7. doi:10.1016/j.futures.2017.05.010.

Saltelli, Andrea, and Mario Giampietro. 2016. What is wrong with evidence based policy ? 1. Science advice at times of crises. Futures: 1-24.

Smil, V. 2013. Should we eat meat? Evolution and consequences of modern Carnivory. Wiley: EBL Ebooks Online.

Struik, Paul C., Thomas W. Kuyper, Lijbert Brussaard, and Cees Leeuwis. 2014. Deconstructing and unpacking scientific controversies in intensification and sustainability: Why the tensions in concepts and values? Current Opinion in Environmental Sustainability 8. Elsevier B.V.: 80-88. doi:10.1016/j.cosust.2014.10.002. 
Tilman, David, Kenneth G. Cassman, Pamela A. Matson, Rosamond Naylor, and Stephen Polasky. 2002. Agricultural sustainability and intensive production practices. Nature 418: 671-677. doi:10.1038 /nature01014.

Tobergte, David R., and Shirley Curtis. 2013. World population prospects the 2015 revision volume I: Comprehensive tables. Journal of Chemical Information and Modeling 53: 1689-1699. doi:10.1017 /CBO9781107415324.004.

Wynne, Brian. 1993. Public uptake of science: A case for institutional reflexivity. Public understanding of science. Vol. 2. SAGE PublicationsSage CA: Thousand oaks, CA. doi:10.1088/0963-6625/2/4/003. 